\title{
Squark/gluino searches in leptonic channels with the CMS experiment
}

\section{Artur Lobanov, on behalf of the CMS Collaboration}

Deutsches Elektronen-Synchrotron (DE)

E-mail: artur.lobanovacern.ch

\begin{abstract}
The second run of the LHC at the increased centre-of-mass energy of $13 \mathrm{TeV}$ significantly extends the sensitivity to possible models of supersymmetry; the largest increase in cross section is expected for heavy gluino and squark pair production. Leptonic final states provide a complementary method to all-hadronic inclusive searches, while also allowing to explore more complicated SUSY scenarios. Several searches performed at the CMS experiment using the 2015 data with an integrated luminosity of $2.3 \mathrm{fb}^{-1}$ are presented. The results are interpreted within the framework of simplified models, and allow to impose limits on a large variety of signal scenarios.
\end{abstract}

Fourth Annual Large Hadron Collider Physics

13-18 June 2016

Lund, Sweden 


\section{Introduction}

One of the most promising theories beyond the Standard Model (SM) is supersymmetry (SUSY), which introduces an additional space-time symmetry between fermionic and bosonic states [1]. Provided the superpartners of the SM particles are not too heavy and $R$-parity is conserved, SUSY offers intriguing solutions to several shortcomings of the SM, including the hierarchy problem and the origin of dark matter.

Given the complexity of the parameter space of any supersymmetric mode $1^{1}$, the framework of simplified model is adopted for many searches of new particles. In such models only the parameters related to the process of interest are included: the superpartner masses, spins and decay branching ratios. Searches for supersymmetry have been already performed for several decades as well as are currently undergoing at the Large Hadron Collider (LHC) by the ATLAS and CMS collaborations. However, the first run of the LHC at centre-of-mass energies of 7 and $8 \mathrm{TeV}$ with large data samples corresponding to about 5 and $20 \mathrm{fb}^{-1}$, respectively, does not show any hint of new physics beyond the SM [2]. Nevertheless, the phase space of interest for SUSY models is not depleted, and the second run of the LHC at the increased centre-of-mass energy of $13 \mathrm{TeV}$ allows to extend these searches even further.

A comparison of the pair-production cross sections for $\sqrt{s}=8$ and $13 \mathrm{TeV}$ is shown in Fig. 1 (left) for different supersymmetric particles (in the coloured/strong sector) depending on their mass. Overall, the gluino production cross sections are the largest for any given mass. When the change in cross section with increasing centre-of-mass energy is considered as in Fig. 1 (right), no difference between the superparticles is found. Nevertheless, given the constraints from Run 1 searches, gluinos are excluded up to a higher mass than squarks and, therefore, their cross section should increase much stronger, than for the latter.

\section{Searches for gluinos at CMS}

In the following, searches for supersymmetric gluon parters, gluinos, using leptonic final states are presented. Leptons can be produced in a large variety of simplified SUSY models in SM-
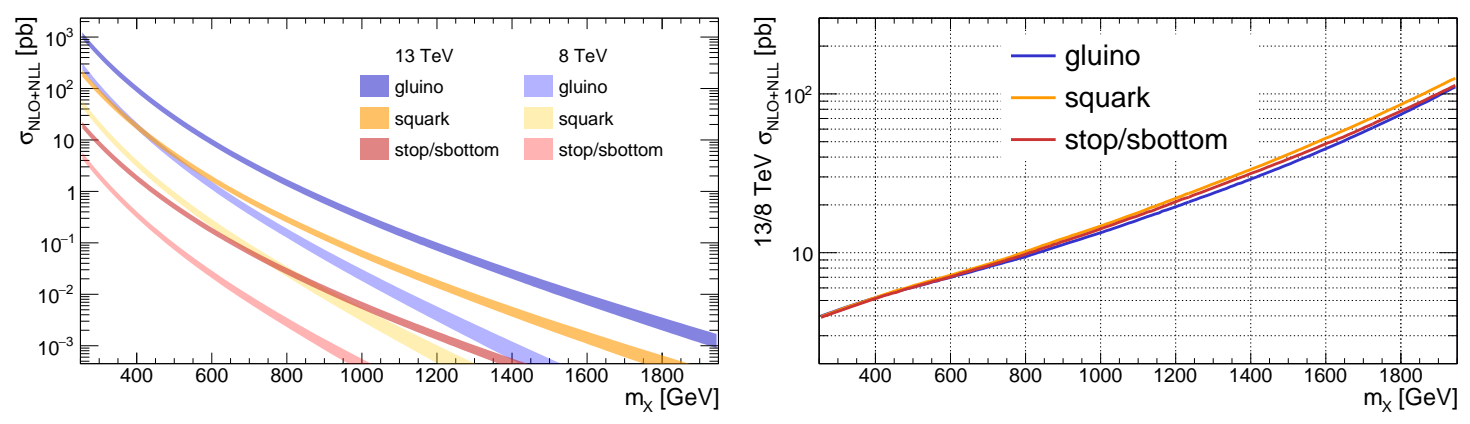

Figure 1: Pair production cross sections for different masses of gluinos, squarks and top/bottom squarks in proton collisions at $\sqrt{s}=8$ and $13 \mathrm{TeV}$ (left) and their respective ratios (right). Based on Ref. [3].

\footnotetext{
${ }^{1}$ The Minimal SUSY extension of the SM (MSSM) incorporates in total 124 parameters.
} 

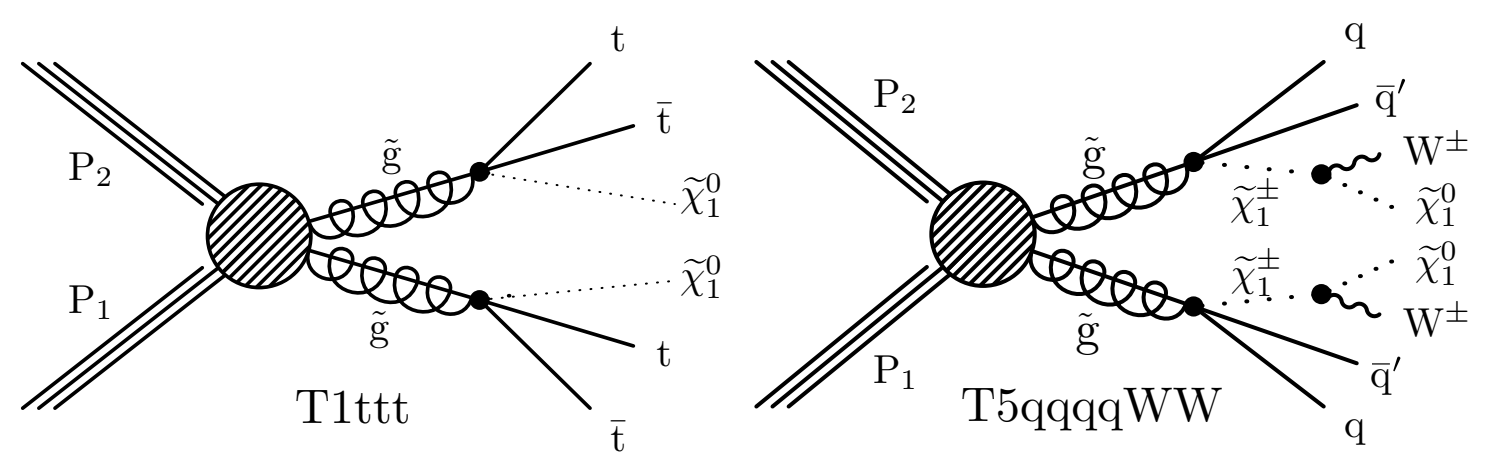

Figure 2: Diagrams for gluino pair production and decays in the simplified models T1tttt (left) and T5qqqqWW (right). The stable neutralino $\widetilde{\chi}_{1}^{0}$ is considered as the lightest supersymmetric particle.

particle decays, e.g. of top quarks and $\mathrm{W}$ or $\mathrm{Z}$ bosons, of which electrons or muons can be efficiently identified in the CMS detector. Therefore, dedicated searches are performed in events with exactly one, two, or more than two of these leptons. For all analyses, multiple search regions are defined and data-driven methods are used to estimate the SM background.

The data for all searches were recorded by the CMS experiment in proton-proton collisions at $\sqrt{s}=13 \mathrm{TeV}$ during Run 2 of the LHC in 2015, corresponding to an integrated luminosity of $2.3 \mathrm{fb}^{-1}$. Despite the almost ten times lower sample size with respect to Run 1, the searches for heavy SUSY particles largely profit from the increased production cross section at $\sqrt{s}=13 \mathrm{TeV}$. A detailed description of the CMS experiment can be found in Ref. [4].

\subsection{Searches with a single lepton}

Searches involving a single isolated lepton are presented in the following. Such final states allow to largely suppress the SM background, while keeping the search still sufficiently inclusive. Figure 2 shows the diagrams of the simplified models T1ttt and T5qqqqWW, which feature production of four top quarks in the former, and four light-flavour quarks together with two $\mathrm{W}$ bosons in the latter. In common are the large number of quarks producing hadronic jets, as well as the presence of two neutralinos - the stable lightest supersymmetric particles (LSP).

\subsubsection{The $\Delta \phi$ search}

The " $\Delta \phi$ search" [5] relies on events with a large jet multiplicity $\left(n_{\text {jet }}\right)$ and hadronic activity, i.e. the scalar sum of the jet momenta $H_{T}=\sum p_{T}$ (jets) $>500 \mathrm{GeV}$, a single isolated hard lepton of $p_{T}>25 \mathrm{GeV}$ and large corresponding leptonic activity $L_{T}=p_{T}$ (lepton) $+E_{T}^{\text {miss }}>250 \mathrm{GeV}$. A veto of b-tagged jets $\left(n_{\mathrm{b}}=0\right)$ allows to enhance the sensitivity to T5qqqqWW models, while the requirement of at least one b-tagged jet is used for the T1ttt search. Further sensitivity to different signal mass scenarios is achieved by splitting the search region into a total of 13 (for $n_{\mathrm{b}}=0$ ) and 30 (for $n_{\mathrm{b}} \geq 1$ ) exclusive categories based on the kinematic variables, respectively.

Signal discrimination is achieved by using the azimuthal angle $\Delta \phi$ between the lepton and Wboson candidate - the vectorial sum of the lepton and $E_{T}^{\text {miss }}$. The main SM backgrounds, dominated by $t \bar{t}+$ jets and $W+$ jets with single leptons in the final state, tend to low angles due to the aligned 

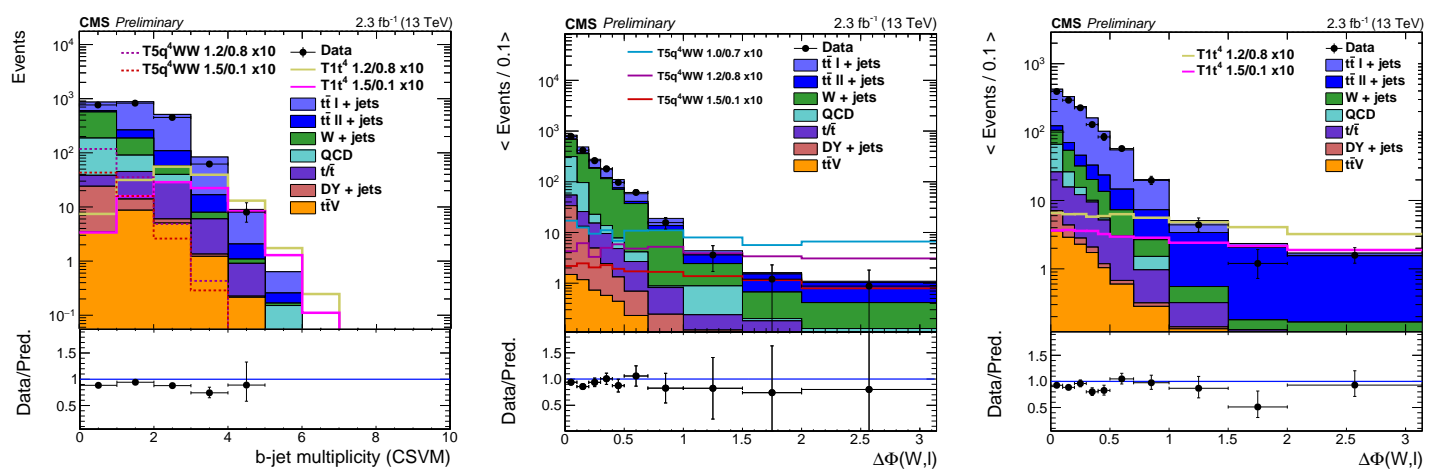

Figure 3: Distributions for the $\Delta \phi$ search after the baseline selection [5]: b-tagged jets multiplicity (left), $\Delta \phi$ for $n_{\mathrm{b}}=0$ (middle) and for $n_{\mathrm{b}} \geq 1$ (right). The simulated SM background samples are stacked, while the SUSY signal distributions are plotted unstacked.

lepton and neutrino-induced $E_{T}^{\text {miss }}$. Signal events from the T1ttt or T5qqqqWW models contain additional missing energy from the escaping LSPs. This leads to a different $\Delta \phi$ shape, allowing to define signal-enriched regions at large angles. Figure 3 (left) shows the multiplicity of b-tagged jets for the major SM backgrounds compared to several benchmark points of the T1tttt and T5qqqqWW signals. The middle and right plots of Fig. 3 illustrate the $\Delta \phi$ distributions with $n_{\mathrm{b}}=0$ and $n_{\mathrm{b}} \geq 1$ requirements, respectively.

The remaining contribution from SM backgrounds is estimated using data-driven methods. Low-to-high $\Delta \phi$ transfer factors are measured in data using low $n_{\text {jet }}$ sidebands. Residual differences between the sidebands and high $n_{\text {jet }}$ search regions are compensated based on correction factors determined in simulation. The minor contribution from QCD multijet events in the low $\Delta \phi$ regions is estimated from orthogonal control samples with looser lepton identification and isolation requirements.

Overall, the background predictions are found to be compatible with the observed data, and therefore, exclusion limits are set based on the considered signal models. This interpretation is discussed in Section 4.

\subsubsection{The $M_{J}$ search}

This search [6] follows a similar methodology as the $\Delta \phi$ search, except that the selected electron or muon is required to have a $p_{T}$ above $20 \mathrm{GeV}$, and a fixed threshold is imposed on the missing transverse energy $E_{T}^{\text {miss }}>200 \mathrm{GeV}$. In addition, only events with at least one b-tagged jet are considered targeting the T1tttt signal. With such a selection the contamination of QCD multijet events is negligible and the SM background is dominated mostly by $t \bar{t}+$ jets events.

The signal discrimination is provided by the commonly used transverse mass variable $m_{T}$ together with the scalar sum of the large-R jet masses $M_{J}$, which is used here for the first time in a SUSY search at the LHC. For the dominating $t \bar{t}+$ jets background $M_{J}$ shows a sharp cut-off at $\approx 2 \times m_{t}$ in absence of initial-state radiation (ISR). The ISR effectively smears out the cut-off, but still allows to discriminate the signal due to the intrinsic large jet multiplicity from four top quarks. In order to increase the sensitivity, a total of 10 exclusive search regions are defined using the 

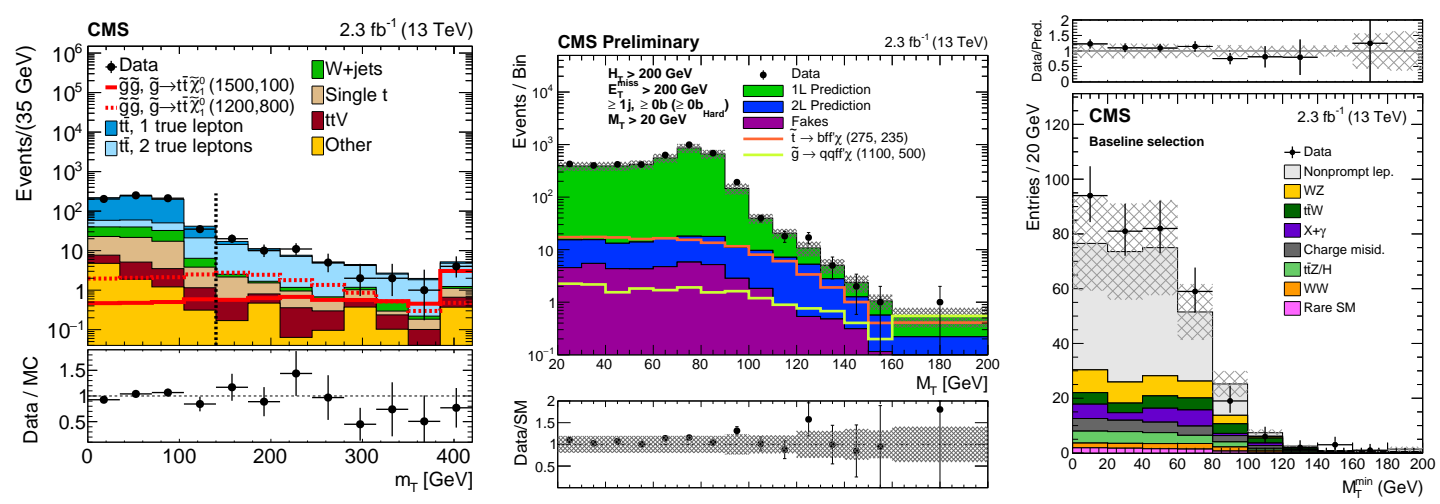

Figure 4: Distributions of the transverse mass for the $M_{J}$ search [6] (left), soft lepton search [7] (middle) and same-sign dilepton search [8] (right).

$n_{\mathrm{jet}}, n_{\mathrm{b}}$ and $E_{T}^{\text {miss }}$ variables. Figure 4 (left) shows the $m_{T}$ distribution inclusively after the baseline selection.

The data-driven background estimation is based on the fact, that the $m_{T}$ and $M_{J}$ variables are mostly uncorrelated for the SM backgrounds. This allows to exploit a similar matrix method as in the $\Delta \phi$ search by defining signal- and background-enriched regions with large and low $m_{T}$ for which the transfer factor can be measured in a background-dominated sideband with low $M_{J}$.

Finally, a multi-bin fit is performed to obtain the prediction in each search category. No significant deviations from the SM are observed, and, hence, limits are set on the T1tttt model.

\subsection{Soft lepton search}

In contrast to the aforementioned searches with energetic leptons, the soft lepton search [7] focuses on events with a single soft lepton of $5<p_{T}<20 \mathrm{GeV}$. This selection allows the search to become sensitive to SUSY scenarios with compressed spectra, i.e. small mass splitting between the massive mother and daughter particles. In case of the considered T5qqqqWW model, $\Delta m\left(\widetilde{\chi}_{1}^{ \pm}, \widetilde{\chi}_{1}^{0}\right)$ is set to $20 \mathrm{GeV}$.

Here, the SM background is suppressed by vetoing events with second leptons or isolated tracks and additional requirements such as $H_{T}>200 \mathrm{GeV}, E_{T}^{\text {miss }}>200 \mathrm{GeV}$ and $m_{T}>20 \mathrm{GeV}$. Search regions are defined using these kinematic variables as well as the multiplicity of jets and b-tagged jets. The $m_{T}$ distribution after the baseline selection is shown in Fig. 4 (middle).

The SM expectation is estimated following different data-driven methods based on the origin of the background. Processes with a single real lepton are estimated from control regions based on events with a single high- $p_{T}$ muon and low $E_{T}^{\text {miss }}$. Dileptonic backgrounds with a lost lepton are estimated from dilepton control regions similar to the signal regions. The remaining background stems from events with no prompt leptons, and is estimated using a tight-to-loose ratio method.

The combined estimates are found to be in agreement with the observation, and, therefore, exclusion limits are obtained on the signal models. 


\section{Searches with two or more leptons}

Final states with two leptons provide more information about the event through various kinematic variables. Therefore, such searches are sensitive to much more specific details of the signal models, and in case of discovery, would allow to measure particular parameters of the new theory. In this section two searches with dilepton events and one search with at least three leptons are presented.

\subsection{Same-sign dilepton search}

The same-sign dilepton search [8] focuses on events with exactly two same-sign charge leptons (electrons or muons), accompanied by large hadronic activity $H_{T}>500 \mathrm{GeV}$ and some missing transverse energy $E_{T}^{\text {miss }}>50 \mathrm{GeV}$. Given the long decay chains of SUSY events, the independent branches may lead to two same-sign leptons in the final state, which is not common in the SM. As a result, the major backgrounds arise from non-prompt leptons and rare SM processes as diboson or $t \bar{t}+$ jets associated boson production.

Signals are discriminated by using the $m_{T}$ variable from the lepton, which minimizes this mass (Fig. 4, right), and search categories are defined also based on the $H_{T}, E_{T}^{\text {miss }}, n_{\text {jet }}$ and $n_{\mathrm{b}}$ variables. In addition, these categories are split based on the $p_{T}$ range of the leptons.

The background sources are estimated in corresponding categories based on data control regions. Backgrounds with non-prompt leptons are predicted based on events, where one of the leptons fails the selection requirements. Opposite-sign dilepton data samples are used to estimate the backgrounds arising from charge misidentification. Simulation is used only to predict the contribution from rare same-sign dileptonic SM processes.

The final predictions are found to be consistent with the observation, thus limits are obtained on various simplified SUSY models as shown in Sec. 4.

\subsection{Multilepton search}

The search presented in Ref. [9] focuses on events containing at least three leptons with a $p_{T}$ down to $10 \mathrm{GeV}$. With such a strict selection, the thresholds on $H_{T}$ and $E_{T}^{\text {miss }}$ can be kept low at 60 and $50 \mathrm{GeV}$, respectively, yielding a high sensitivity to the signal models. Search categories are defined further based on $H_{T}, E_{T}^{\text {miss }}, n_{\mathrm{b}}$ and on the presence or absence of a lepton pair compatible with the $\mathrm{Z}$ boson.

Like in the same-sign dilepton analysis, the remaining SM backgrounds are estimated in categories corresponding to their origin. Non-prompt lepton processes are estimated as well from loose-lepton samples. Diboson and other rare SM backgrounds are taken from simulation using normalization factors obtained in data sidebands.

No significant excess is observed with respect to the SM background prediction, allowing to derive limits on the considered SUSY models.

\subsection{Opposite-sign dilepton search}

The search in final states with two opposite-sign, same-flavour leptons [10] targets signal models with two leptons produced in a single decay branch of one of the SUSY particles. Two different signal topologies are considered leading to independent approaches. 
Final states with a $\mathrm{Z}$ boson are used to search for resonant-like excesses compatible with the Z-boson mass. For models with the second-to-lightest neutralino $\widetilde{\chi}_{2}^{0}$ decaying to the $\widetilde{\chi}_{1}^{0}$ through an intermediate slepton $\tilde{\ell}$ as $\widetilde{\chi}_{2}^{0} \rightarrow \widetilde{\ell} \ell \rightarrow \widetilde{\chi}_{1}^{0} \ell \ell$, a kinematic edge is expected in the invariant mass of the lepton pair $\left(m_{\ell \ell}\right)$.

The search categories for both searches are based on $m_{\ell \ell}$, the jet and b-tagged jet multiplicities, $H_{T}$ and missing transverse energy $E_{T}^{\text {miss }}$. In addition, a special signal search region is included to test the $3 \sigma$ excess observed by ATLAS in the Run 1 data [11].

The SM backgrounds, dominated by $t \bar{t}+$ jets and $Z+$ jets production, are estimated from data in two categories. Contributions from flavour symmetric processes are estimated using oppositeflavour control regions, while Drell-Yan-like background predictions are obtained from $E_{T}^{\mathrm{miss}}$ templates measured in $\gamma+$ jets control samples.

No significant excess is observed in any of the search categories.

\section{Results}

A multitude of searches for supersymmetry in the leptonic channels has been presented. Unfortunately, no hints of physics beyond the Standard Model have been observed. This allows to impose exclusion limits on certain signal scenarios using the aforementioned simplified models. In the following, limits for the most common models are presented. The interested reader can find additional interpretations in the references of the individual analyses and in Ref. [12].

Figure 5 (left) shows the cross section limits at $95 \% \mathrm{CL}$ as a function of the gluino and neutralino masses, as obtained by the $\Delta \phi$ search for the T1ttt model. A comparison of this limit to other analyses is shown in Fig. 5 (right). Overall, the single-lepton searches achieve the strongest exclusion, with the $\Delta \phi$ search being more sensitive in the compressed region where neutralinos below $800 \mathrm{GeV}$ are excluded, and the $M_{J}$ search excluding gluinos below $1575 \mathrm{GeV}$ for low neutralino masses.

In case of the T5qqqqWW model, different mass splittings between the chargino and neutralino are considered for interpretation depending on the search. The soft-lepton search limit for the compressed scenario is shown in the left plot of Fig. 6, while the right plot shows the summary of limits from the various analyses targeting the less compressed scenario. Also in this case the single-lepton searches show the strongest exclusion power, however, the two- and multilepton analyses are more sensitive to complex topologies not considered here.

\section{Conclusion}

The searches for SUSY performed by CMS presented here were based on data recorded during the very beginning of the LHC Run 2. No hints of physics beyond the Standard Model were observed at the new centre-of-mass energy of $13 \mathrm{TeV}$. Already at this point, with only $2.3 \mathrm{fb}^{-1}$ of integrated luminosity, many Run 1 results have been significantly outperformed given the increased production cross sections. The remaining bulk of Run 2 data will allow to explore even further the validity of the SM and continue the search for supersymmetry. 

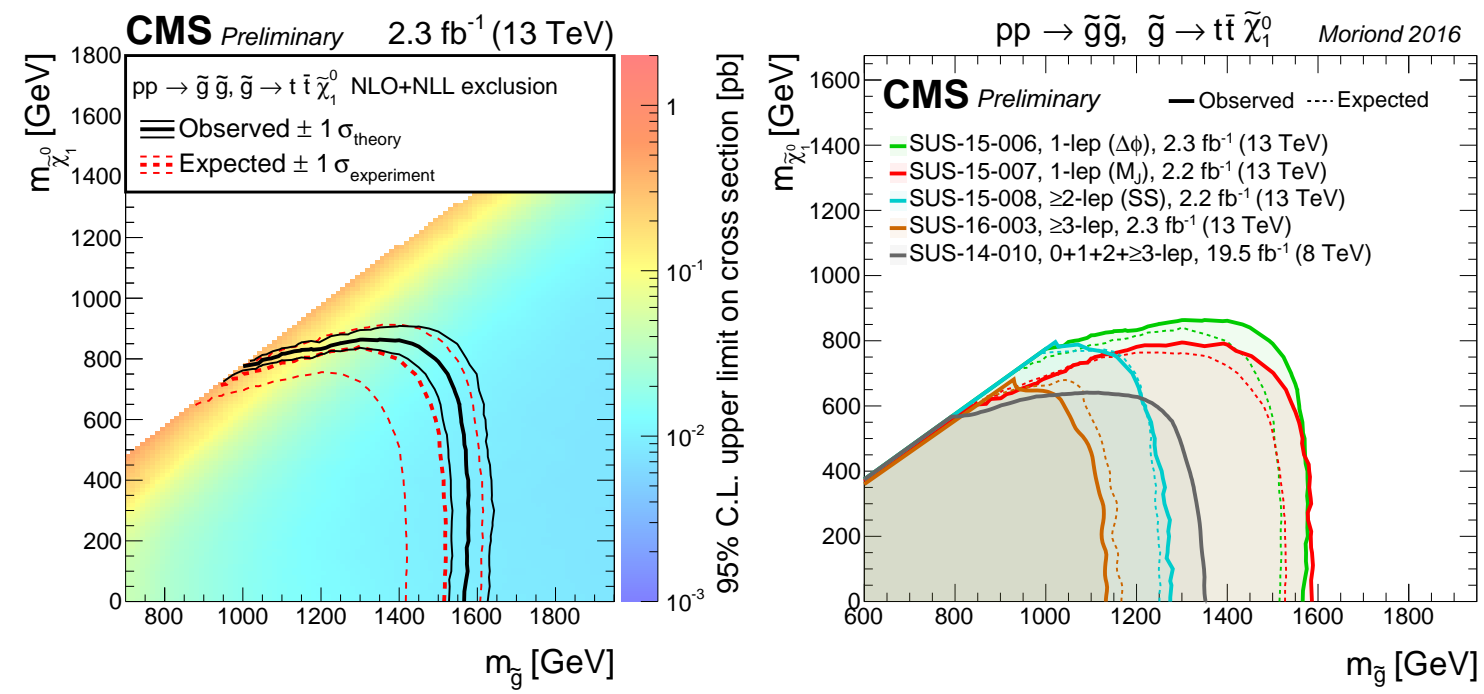

Figure 5: Left: cross section limits at $95 \% \mathrm{CL}$ on the $\mathrm{T} 1 \mathrm{tttt}$ model as a function of the gluino and neutralino (LSP) masses, as obtained by the $\Delta \phi$ search. The black and red lines indicate the observed and expected exclusion contours, respectively. Right: T1ttt exclusion contours as obtained by different searches in CMS.
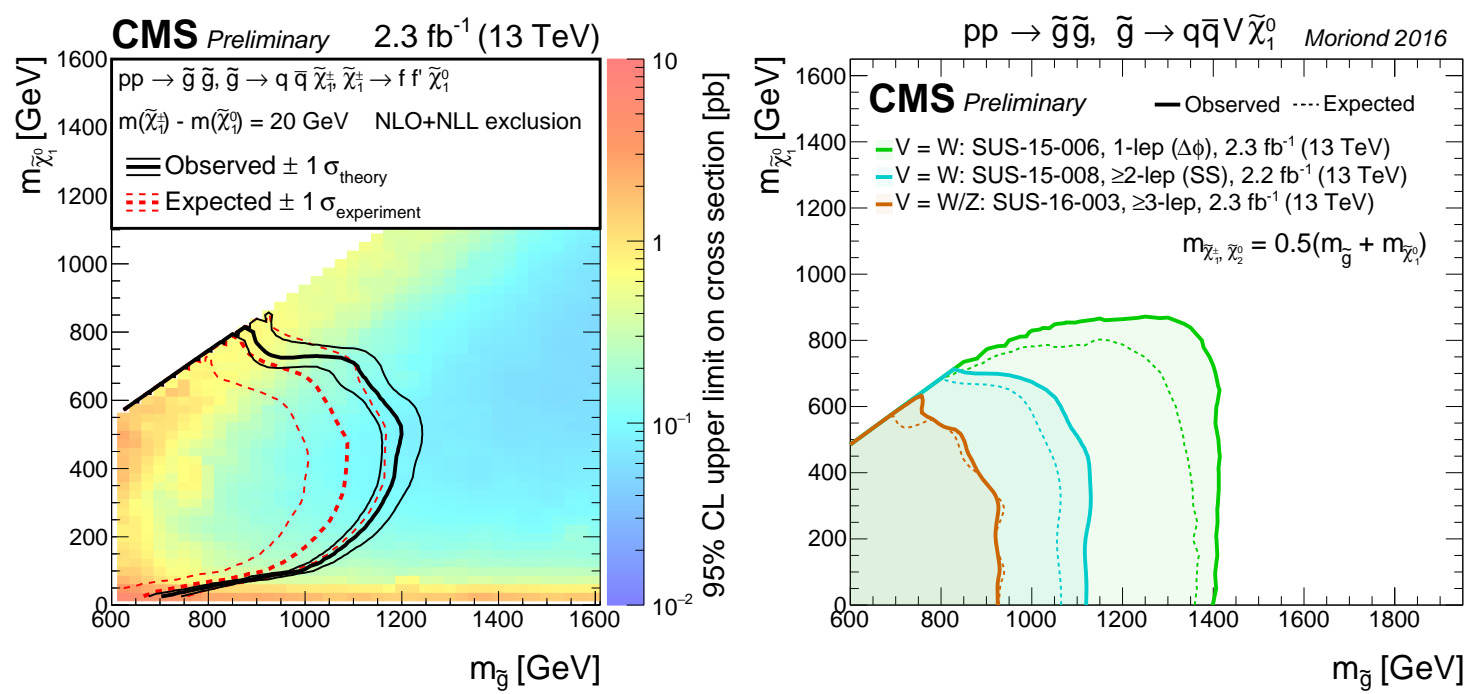

Figure 6: Left: cross section limits at $95 \% \mathrm{CL}$ on the T5qqqqWW model as a function of the gluino and neutralino (LSP) masses, as obtained by the soft-lepton search. Right: exclusion contours as obtained by different CMS searches within the T5qqqqWW model. 


\section{References}

[1] S. P. Martin, A Supersymmetry primer, Adv. Ser. Direct. High Energy Phys. 21 (2010) 1 [hep-ph/9709356].

[2] I. Melzer-Pellmann and P. Pralavorio, Lessons for SUSY from the LHC after the first run, Eur. Phys. J. C 74 (2014) 2801 doi:10.1140/epjc/s10052-014-2801-y [arXiv:1404.7191 [hep-ex]].

[3] LHC SUSY Cross Section Working Group, https://twiki.cern.ch/twiki/bin/view/LHCPhysics/SUSYCrosssections

[4] S. Chatrchyan et al. [CMS Collaboration], The CMS experiment at the CERN LHC, JINST 3, S08004 (2008). doi:10.1088/1748-0221/3/08/S08004

[5] CMS Collaboration, Search for supersymmetry in events with one lepton in proton-proton collisions at $\operatorname{sqr}(s)=13 \mathrm{TeV}$ with the CMS experiment. CMS-PAS-SUS-15-006.

[6] CMS Collaboration, Search for supersymmetry in pp collisions at $\sqrt{s}=13 T e V$ in the single-lepton final state using the sum of masses of large-radius jets, Submitted to JHEP [hep-ex/1605.04608].

[7] CMS Collaboration, Search for new physics in the one soft lepton final state using 2015 data at $\sqrt{s}=13 \mathrm{TeV}, \mathrm{CMS}-\mathrm{PAS}-\mathrm{SUS}-16-011$.

[8] V. Khachatryan et al. [CMS Collaboration], Search for new physics in same-sign dilepton events in proton-proton collisions at $\sqrt{s}=13 \mathrm{TeV}$, Eur. Phys. J. C 76, no. 8, 439 (2016) doi:10.1140/epjc/s10052-016-4261-z [arXiv:1605.03171 [hep-ex]].

[9] CMS Collaboration, Search for SUSY with multileptons in $13 \mathrm{TeV}$ data, CMS-PAS-SUS-16-003.

[10] V. Khachatryan et al. [CMS Collaboration], Search for new physics in final states with two opposite-sign, same-flavor leptons, jets, and missing transverse momentum in pp collisions at $\sqrt{s}=$ $13 \mathrm{TeV}$, [arXiv:1607.00915 [hep-ex]].

[11] G. Aad et al. [ATLAS Collaboration], Search for supersymmetry in events containing a same-flavour opposite-sign dilepton pair, jets, and large missing transverse momentum in $\sqrt{s}=8 \mathrm{TeV}$ pp collisions with the ATLAS detector, Eur. Phys. J. C 75, no. 7, 318 (2015) Erratum: [Eur. Phys. J. C 75, no. 10, 463 (2015)] doi:10.1140/epjc/s10052-015-3661-9, 10.1140/epjc/s10052-015-3518-2 [arXiv:1503.03290 [hep-ex]].

[12] CMS Collaboration, Further SUSY Simplified Model interpretations for Moriond 2016, CMS-PAS-SUS-16-004. 